\title{
Improvement of the Approach Vehicle Detection by the Supersonic Wave
}

\author{
Kosuke Kuwayama, Yuto Tanaka, Yoshihisa Nakatoh \\ Kyushu Institute of Technology, 1-1 Sensui-cho, Tobata-ku, Kitakyushu 804-8550, Japan \\ *Corresponding Author: p349416k@mail.kyutech.jp
}

\begin{abstract}
As the population ages, and the traffic environment surrounding elderly people is danger. The rate of elderly people falling victim to fatal traffic accidents has become more than $50 \%$. We can prevent traffic accidents if we detect that the approaching vehicle may collide with the walking elderly person. Therefore, we considered that it would be useful if we could build a system to inform us of the approaching vehicle by a supersonic wave ${ }^{(1)}$. In this paper, we have considered the case when there is an angle between a pedestrian and parametric loud speaker, and the difference of the number of speaker elements to improve this system. As a result, the angle had a low influence on detection rate in $50 \mathrm{~m}$. In contrast, the scale of the speaker had a high influence.
\end{abstract}

Keywords: supersonic wave, parametric loudspeaker, vehicle detection

\section{Introduction}

As the population ages, and the traffic environment surrounding elderly people is danger ${ }^{(2)}$. The rate of elderly people falling victim to fatal traffic accidents has become more than $50 \%$. As for the traffic accidents involving elderly people, a delay in the drop of the athletic capability due to aging and dangerous evasion movement is regarded as a factor ${ }^{(3-4)}$. If elderly people can perceive the approaching vehicle at an earlier stage, they can prevent being involved in a traffic accident. Therefore, we considered that it would be useful if we could build a system to inform us of approaching vehicle by a supersonic wave.

In general, millimeter-wave radar and infrared radar is that electric wave and light wave beamed from vehicle side. We considered that electric wave and light wave can use for vehicle detection, if pedestrian can receive the waves beamed from vehicle side. However, vehicle radar and electric power saving millimeter-wave radar as DSSS

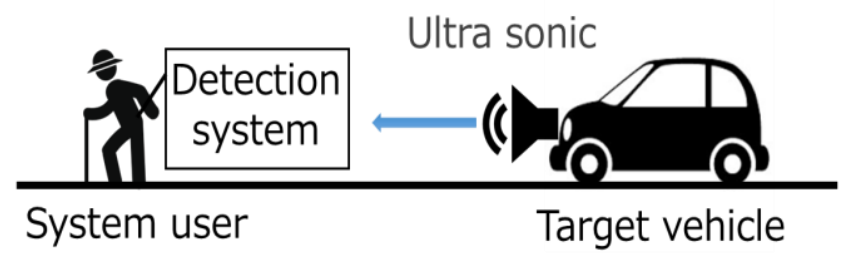

Fig.1 Image of vehicle detection system

(Driving Safety Support Systems) is subject to use by radio wave method, and it is difficult to get parts for the radars. In contrast, supersonic wave can use not to be subject by radio wave method. However, we should entertain influence to human being. As previously described, this system use supersonic wave for vehicle detect. In this paper, we have considered the case when there is an angle between a pedestrian and speaker, and the size of the speaker has changed to improve this system. We simulate that there is a pedestrian aside of a road $3 \mathrm{~m}$ wide, and performed detection experiment in the range of $45 \mathrm{~m}$ from $5 \mathrm{~m}$ under the environment of a background noise of $45 \mathrm{dBA}$. This experiment are performed with big parametric loud speaker (101 elements). Moreover, we used a smaller parametric loud speaker (13 elements), and performed detection experiment in the same place as the foregoing experiment.

\section{System Overview}

Figure 1 is the overview of this system. We attached a supersonic wave loudspeaker to the side of the vehicle. Figure 2 is a block diagram of transmission mechanism. Attached supersonic wave loudspeaker sends a supersonic wave to detect vehicles. Figure 3 is a block diagram of the receiver side. Walker received the wave using a broadband microphone on the sidewalk. Received supersonic waves are converted into the audible range. When converted signals turned into background noise level $+3 \mathrm{~dB}$, walker stated that they were able to detect the approaching vehicles. 


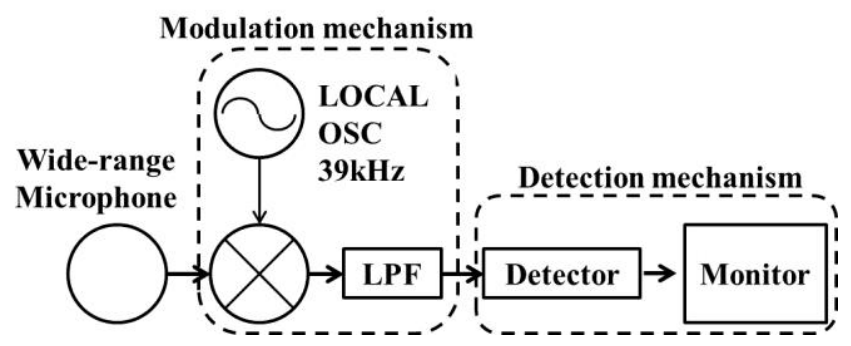

Fig. 2 Brock diagram of the transmission side

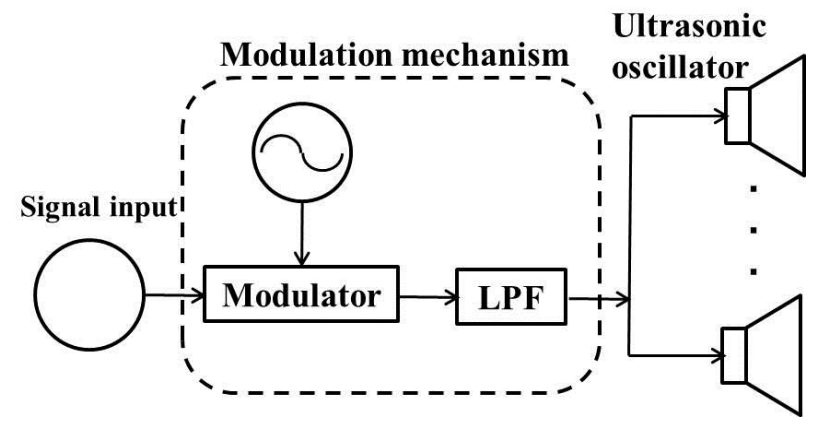

Fig. 3 Brock diagram of the receiver side

\subsection{Parametric Loudspeaker}

In general, a plurality of side-by-side unit of the ultrasonic transducer, it is called parametric loudspeaker. Ultrasonic waves are generated at an angle of a few degrees from the parametric loudspeaker. Ultrasonic wave shows straight and very little spread around. Seeing from a point of view called the use to our life, the supersonic wave does not cause radiation damage, but influence by the distance decrement is very high. Therefore, we thought to overcome the problem of the distance decrement by using the parametric loudspeaker which formed a very high directivity.

\subsection{Transmission Mechanism}

The transmission mechanism uses a parametric loudspeaker (it carries AT40-10PB3 made by Nippon Ceramic, big one has 101 elements and small one has 13 elements). Figure 2 is a Brock diagram of the transmission mechanism. The modulation device uses a supersonic carrier wave as $40 \mathrm{kHz}$, located at an irregular time of $31.5 \mathrm{~Hz}$ by a cover abnormality signal (a band pulse). Because the device makes detection easier and has less influence on human beings, this system uses a band pulse concentrated in terms of time.

\subsection{Reception Mechanism}

Figure 3 is a block diagram of the receiver side. At first, the supersonic wave are converted into the audible range by mixing the received information in a broadband microphone with a local oscillation, and then passes through a low-path filter (Heterodyne method). We consider that we could detect an approaching vehicle when converted signals are turned into the background noise level of $+3 \mathrm{~dB}$ in the supersonic wave domain.

\section{Input Waveform to the Parametric Loudspeaker}

The IRPA established the regulation that is located in Table 1 for international permission value about the supersonic wave domain revelation, and there is a description that $3 \mathrm{~dB}$ may raise a permission level if revelation time becomes half ${ }^{(5-7)}$. According to this regulation, we set the sound pressure level at $109 \mathrm{~dB}$ (distance $1 \mathrm{~m}$ from the parametric loudspeaker) in order not to influence hearing ability by the revelation. In order to the device makes detection easier and has less influence on human beings, we uses a band pulse concentrated in terms of time. Figure 5 is the waveform of band pulse. Pulse is that of the signal to a steep change in a short period of time. In other words, the signal change is sudden presence only at short time in general terms. We have used the $1 / 1$ Octave band pulse at $31.5 \mathrm{~Hz}$.

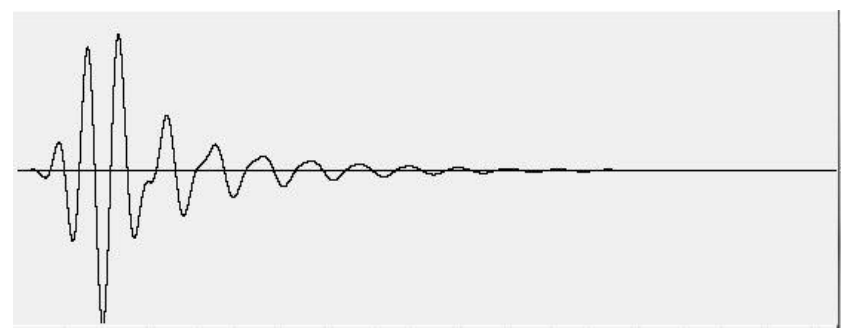

Fig. 5 Waveform of band pulse

Table 1. Aerial Supersonic Permissible Level

\begin{tabular}{|c|c|}
\hline $\begin{array}{c}\text { Center of the } 1 / 3 \text { Oct. } \\
\text { band }[\mathrm{kHz}]\end{array}$ & $\begin{array}{c}\text { Sound pressure level } \\
{[\mathrm{dB}]}\end{array}$ \\
\hline 20 & 75 \\
\hline 25 & 110 \\
\hline 31.5 & 110 \\
\hline 40 & 110 \\
\hline 50 & 110 \\
\hline
\end{tabular}




\section{Influence of the Background Noise}

In previous experiment, we recorded the background noise of three patterns, a quiet environment (45dBA), an uncrowded street (55dBA), and a crowded street (65dBA) to check the influence of the background noise in the supersonic wave domain. After recording, we calculated power from waveform of the background noise in the supersonic wave domain $(39-41 \mathrm{kHz})$. As a result, a change in the spectral envelope waveform was only seen in cases where a vehicle or a bicycle passed beside us (2-3m). Therefore, we found that there is almost no influence in any condition by the background noise.

\section{Detection Performance}

In previous experiment, there is almost no influence in any condition by the quiet environment of background noise of $45 \mathrm{dBA}$. Therefore, we simulate that there is a pedestrian aside of a road $3 \mathrm{~m}$ wide, and performed detection experiment in the range of $45 \mathrm{~m}$ from $5 \mathrm{~m}$ under the environment of a background noise of $45 \mathrm{dBA}$. This experiment are performed with big parametric loud speaker (101 elements). Moreover, we used a smaller parametric loud speaker (13 elements), and performed detection experiment in the same place as the foregoing experiment. Table 2 is experimental conditions.

Table 2. Experimental condition

\begin{tabular}{|c|c|}
\hline Microphone & $\begin{array}{c}\text { SPM0404UD5 } \\
\text { (made by KNOWLES } \\
\text { ACOUSTICS) }\end{array}$ \\
\hline $\begin{array}{l}\text { Microphone } \\
\text { position }\end{array}$ & $60 \mathrm{~cm}$ from the ground \\
\hline $\begin{array}{l}\text { Parametric } \\
\text { loudspeaker }\end{array}$ & $\begin{array}{c}\text { AT40-10PB3 } \\
\text { ( made by Nippon Ceramic) }\end{array}$ \\
\hline $\begin{array}{c}\text { Sound pressure } \\
\text { level } \\
\text { (Distance } 1 \mathrm{~m} \text { ) }\end{array}$ & $\begin{array}{c}109 \mathrm{~dB} \text { (101 elements) } \\
80 \mathrm{~dB} \text { (13 elements) }\end{array}$ \\
\hline Input signal & Band pulse $(31.5 \mathrm{~Hz}, 0.74 \mathrm{sec})$ \\
\hline Weather & Sunny \\
\hline Temperature & $27{ }^{\circ} \mathrm{C}$ \\
\hline Wind & $0 \mathrm{~m} / \mathrm{s}$ \\
\hline $\begin{array}{c}\text { Background } \\
\text { noise }\end{array}$ & $45 \mathrm{dBA}$ \\
\hline Number of trials & For each 100 times \\
\hline
\end{tabular}

5.1 Results of the Case When There is an Angle Between a Pedestrian and Parametric Loudspeaker

Figure 6 is a picture of when we performed a detection experiment on the assumption that there is a pedestrian aside of road. The width of the road that we have used in the experiment was a $3 \mathrm{~m}$, and there are not preventives around the road. Figure 7 shows the spectrum of recorded signal and the rate of detection when we set the background noise level to $+3 \mathrm{~dB}$ for the threshold at $40 \mathrm{kHz}$. After analyzing the spectrum of recorded signal, a peak was seen at around 500$600 \mathrm{~Hz}$ (Relative value of $40 \mathrm{kHz}$ ) in any distance (angle). And we can found that the amplitude decreased with the increasing distance from parametric loudspeaker. However, as a result, the detection rate at $45 \mathrm{~m}$ was the more than $90 \%$.

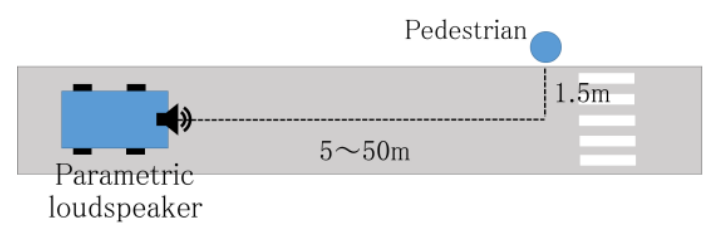

Fig. 6 Simulation there is a pedestrian aside of road

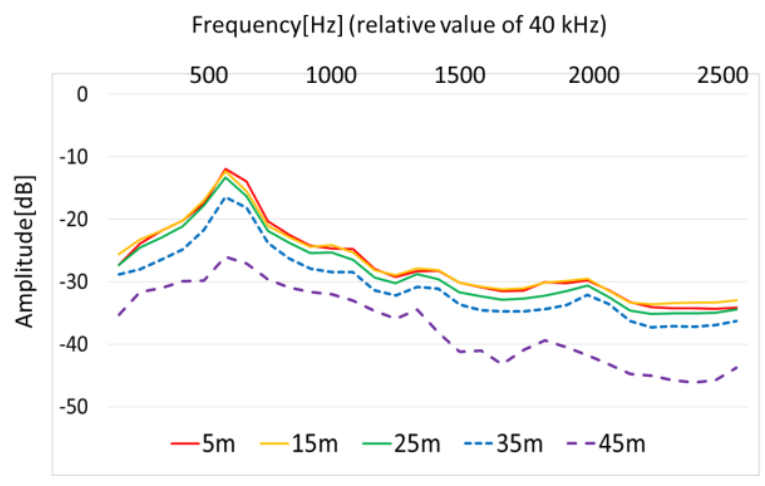

(a) Spectrum

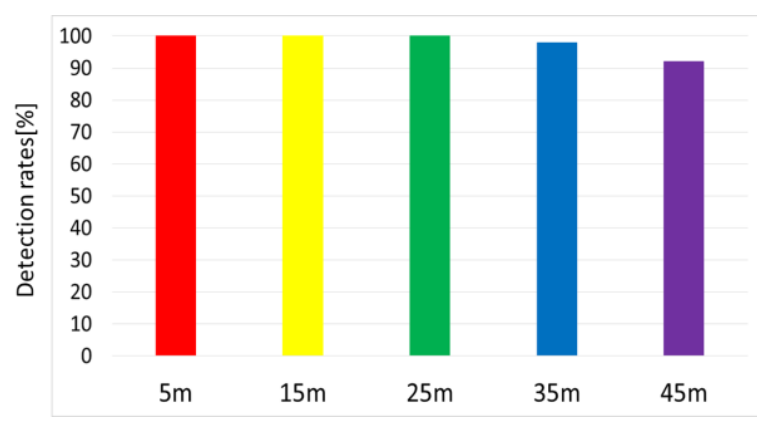

(b) Number of trials 100 times

Fig. 7 The case when there is angle between a pedestrian and speaker 


\subsection{Results of the Case When the Size of Speaker Has Changed}

This experiment was performed in the same place as the foregoing experiment. Figure 8 is a picture of 2 parametric loudspeaker were used in this experiment. The left loudspeaker consists of 101 speaker elements, and right one consists of 13 speaker elements. Figure 9 shows results of detection experiment at $35 \mathrm{~m}$ (direct distance). We can found that a peak of spectrum and rate of detection decrease with the smaller loudspeaker

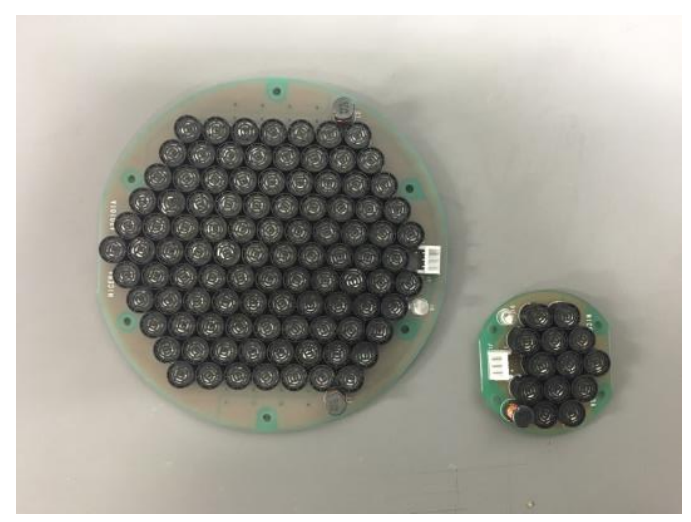

Fig. 8 The size of 2 parametric speakers

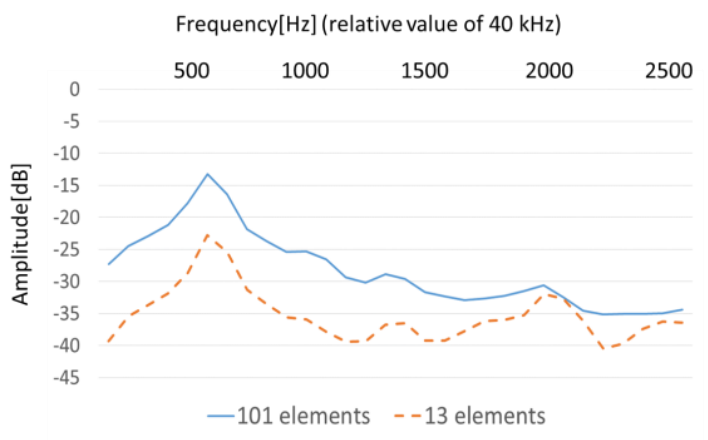

(a) Spectrum

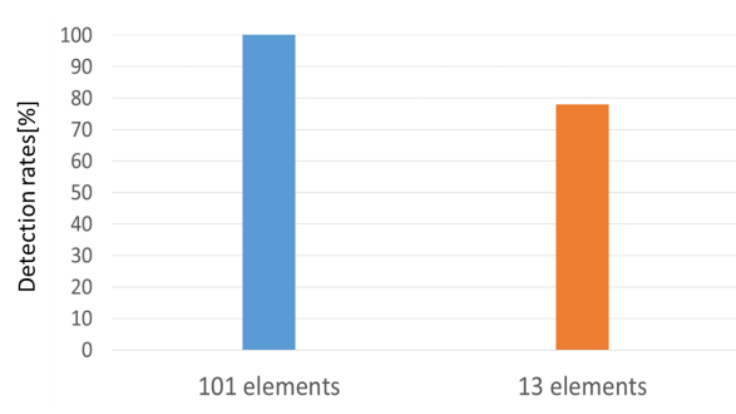

(b) Number of trials 100 times

Fig. 9 The case of 101 and 13 speaker elements

\section{Conclusions}

In this paper, we performed validation about pedestrian angle and size of speaker, in addition to the approach vehicle detection by straight-line as previous study. In the range of $5-45 \mathrm{~m}$, the influence to detection rate by angle between a parametric loudspeaker and a pedestrian was not present. And, in a pedestrian aside of a road, it is considered that detection rate is dependent on distance between a parametric loudspeaker and a microphone. By contrast, the detection rate decreased by $20 \%$ when the size of a parametric loudspeaker has changed. We are going to perform experiments with variously sized speaker and should judge practical and commercial the number of speaker elements. Additionally, we need to produce computer programs for the supersonic detection.

\section{References}

(1) Yoshihiro Furuno, Yuto Tanaka, Yoshihisa Nakatoh, "Study of the approach vehicle detection by the supersonic wave" Proceedings of the $3 \mathrm{rd}$ IIAE International Conference on Intelligent Systems and Image Processing 2015, pp309-312, 2015

(2) National Police Agency in Japan, " The outbreak situation of a traffic accident in 2012", 2012

(3) Tatsuro Mitsui, "Relations of body function and the traffic fatal accident of the elderly person", Reports of the National Research Institute of Police Science. Research on traffic safety and regulation Vol.36, No. 1, pp. 58-69, 1995

(4) Tsutomu Akiyama, Kazutaka Mitobe, Noboru Yoshimura, Makoto Takahashi, "Study of aged person by Perceptual-motor Coordination", Technical report of IEICE, HCS97-14, pp. 49-56, 1997

(5) Masayoshi Kondo, Toru Yoshida, Hideyuki Nomura, Tomoo Kamakura, "Acoustic characteristics of ear canal model for studying ultrasonic exposure", IEICE Technical Report, US2009-75, pp. 25-28, 2009

(6) Kan-ya Ito, Hideyuki Nomura, Tomoo Kamakura, "High-frequency Audiometry under Presentation of ultrasound", IEICE Technical Report, US2011-61, pp.19-22, 2011

(7) IRPA/WHO, Ultrasound, "Interim guidelines on limits of human exposure to airborne ultrasound", Health Phys., 46, pp. 969-974, 1984 